\title{
BMJ open Reducing risk with e-based support for adherence to lifestyle change in hypertension (REACH): protocol for a multicentred randomised controlled trial
}

\author{
Robert P Nolan, ${ }^{1,2}$ Sam Liu, ${ }^{1,2}$ Ross Feldman, ${ }^{3}$ Martin Dawes, ${ }^{4}$ Susan Barr, ${ }^{5}$ \\ Hazel Lynn, ${ }^{6}$ Femida Gwardy-Sridhar, ${ }^{3}$ Scott G Thomas, ${ }^{7}$ Jack Goodman, ${ }^{7}$ \\ Paul Oh, ${ }^{8}$ Janusz Kaczorowski, ${ }^{9}$ Caroline Chessex, ${ }^{10}$ Vladimir Hachinski, ${ }^{11}$ \\ Kevin Shoemaker ${ }^{12}$
}

To cite: Nolan RP, Liu S, Feldman $\mathrm{R}$, et al. Reducing risk with e-based support for adherence to lifestyle change in hypertension (REACH): protocol for a multicentred randomised controlled trial. BMJ Open 2013;3:e003547 doi:10.1136/bmjopen-2013003547

- Prepublication history and additional material for this paper is available online. To view these files please visit the journal online (http://dx.doi.org/10.1136/ bmjopen-2013-003547).

Received 4 July 2013 Accepted 26 July 2013

For numbered affiliations see end of article.

Correspondence to Dr Robert P Nolan; rnolan@uhnres.utoronto.ca

\section{ABSTRACT}

Introduction: Web-based lifestyle counselling designed to improve adherence to self-management behaviours for diet, exercise and medication has been shown to reduce blood pressure (BP). However, the long-term clinical outcome of these interventions is not established. Our aim was to establish whether an e-counselling program is independently associated with improved clinical outcomes over a 12-month period, as defined by the following criteria: (1) reduction of systolic BP, diastolic BP, pulse pressure and associated risk factors for cardiovascular events; and

(2) adherence to self-management behaviour (diet, exercise, smoke-free living and prescribed medication). Methods and analysis: Reducing risk with e-based support for adherence to lifestyle change in hypertension is a two-parallel group, double-blind randomised controlled trial that will utilise a two (Groups: e-counselling vs control) by three (assessment intervals: baseline, 4-month and 12-month outcome) design. BP, lipoprotein cholesterol, physical activity and dietary behaviours and psychological distress will be measured at each assessment. We plan to recruit 528 participants (35-74 years of age) diagnosed with stage 1 or 2 hypertension (systolic BP, 140-180 mm Hg; diastolic BP 90-110 mm Hg) from three major cities (Toronto, London, Vancouver) and one rural area (Grey Bruce region) across Canada between February 2012 and July 2015. Controls will receive general educational e-messages on heart healthy living and the ecounselling group will receive tailored e-messages that are matched to their stage of readiness for change. For both groups, e-messages will be sent proactively on a weekly basis during months $1-4$, then bi-weekly during months $5-8$ and then monthly during months 9-12.

Ethics and dissemination: Ethical approval has been obtained from all recruitment sites. This will be one of the first studies to evaluate the long-term efficacy of preventive e-counselling strategies for

\section{ARTICLE SUMMARY}

\section{Article focus}

- This article describes the study protocol of a multicentred double-blind randomised controlled trial evaluating the effectiveness of a 12-month lifestyle e-counselling program in reducing risk factors for cardiovascular disease and improving adherence to self-management behaviours in patients with hypertension.

Key messages

- An e-counselling strategy is becoming increasingly prominent in the development of communitybased initiatives for prevention.

- The long-term clinical outcome of these interventions is not established.

- Reducing risk with e-based support for adherence to lifestyle change in hypertension (REACH) will be one of the first studies to evaluate the long-term efficacy of preventive e-counselling for cardiovascular disease prevention in patients with hypertension, and the findings from this study will be used to guide the ongoing development of e-counselling services at the Heart and Stroke Foundation of Canada.

Strengths and limitations of this study

- A key strength of this trial is that the intervention is grounded in a clinical applied theory of behaviour change. Also, an unbiased response by participants to the REACH protocol will be facilitated by frequent and sustained e-messages that are supportive of controls and the Intervention group.

- A limitation of REACH is that any supplementary heart health program in which participants are enrolled during the REACH trial is only controlled statistically, not methodologically. Owing to technical limitations, there is only limited interactivity in the interface between the e-counselling program and participants. 
cardiovascular disease prevention in patients with hypertension. Findings from this study will be used to guide the ongoing development of e-counselling services.

Trial Registration: Clinicaltrial.gov NCT01541540; http:// clinicaltrials.gov/ct2/show/NCT01541540.

\section{INTRODUCTION}

Individuals with hypertension are at increased risk for cardiovascular disease (CVD), stroke, coronary heart disease, heart failure and CVD mortality. ${ }^{1}$ Hypertension is prevalent among $19-21 \%$ of Canadian adults 20-79 years of age, and its prevalence rises to $53 \%$ among adults 60-79 years of age and higher among elderly adults. ${ }^{2}$ Hypertension is treated and controlled among only $66 \%$ of Canadian adults. ${ }^{2} 3$ This is a matter of concern as the risk of cardiovascular mortality among adults 40-69 years of age rises with elevated blood pressure (BP), doubling with each increase of $20 \mathrm{~mm} \mathrm{Hg}$ systolic or $10 \mathrm{~mm} \mathrm{Hg}$ diastolic, from the base index of 115/75 mmHg. ${ }^{4}$

Best practice guidelines in North America ${ }^{5}{ }^{6}$ and Europe $^{7}$ advocate the strategy of combining pharmacotherapy with lifestyle counselling for patients diagnosed with hypertension and/or those who are at elevated risk for CVD. Meta-analyses indicate that programs aimed at improving self-management behaviours significantly decrease systolic BP (SBP) by $3.0-8.7 \mathrm{~mm} \mathrm{Hg}$ relative to controls. $^{8-10}$ A major challenge for a community-based CVD prevention strategy is to extend the reach of therapeutic lifestyle counselling to individuals with hypertension and/or high CVD risk without overtaxing patient care resources. Currently, $73 \%$ of the Canadian population reports personal access to the internet, which includes $61-69 \%$ of Canadians in the two lowest income quartiles, and $61 \%$ of older Canadians (aged 55-64 years). ${ }^{11}$ The use of e-based programs that are designed to improve self-management behaviours for diet, exercise, smoke-free living and medication adherence may be well suited to meet this challenge.

Our previous trials of COHRT ${ }^{12}$ and I-START ${ }^{13}$ have shown that it is feasible to apply an evidence-based model of behavioural counselling with motivational interviewing $^{14}$ to a telehealth or e-based program in order to achieve a reduction in SBP, diastolic BP (DBP) and pulse pressure (PP). This was achieved by means of increased adherence to self-reported diet and exercise, and it was independent of the therapeutic benefit of medications. However, it is not established whether e-counselling improves clinical outcome and adherence over a long-term interval of at least 12 months. Thus, the aim of the reducing risk with e-based support for adherence to lifestyle change in hypertension (REACH) trial is to establish whether an e-counselling program is independently associated with improved clinical outcomes over a 12-month period, as defined by the following criteria: (1) reduction of $\mathrm{BP}$ and associated risk factors for cardiovascular events; and (2) adherence to selfmanagement behaviour for diet, exercise, smoke-free living and prescribed medication.

Our primary hypotheses are that e-counselling (vs control) will significantly reduce (1) SBP, DBP and PP at the 4-month and 12-month assessment intervals, as measured by a validated protocol for automated BP assessment in the clinic; and (2) lipoprotein cholesterol (total, low-density and total/high-density ratio) and the Framingham index of 10-year absolute risk for CVD.

Our secondary hypotheses are that e-counselling (vs control) will significantly increase adherence to selfmanagement behaviours at the 4-month and 12-month outcomes, as measured by the following objective and validated indices: mean 4-day step count recorded by a triaxial pedometer, dietary sodium $\leq 100 \mathrm{mmol} /$ day measured by $24 \mathrm{~h}$ urinary sodium, smoke-free living measured by salivary cotinine, fruit and vegetable intake $\geq 9$ 12 servings/day as measured by the Diet History Questionnaire (DHQ) assessment and physical activity $\geq 150 \mathrm{~min} /$ week as measured by the Recent Physical Activity Questionnaire (RPAQ); and medication adherence as defined by the medication possession ratio (derived from the pharmacy refill data) among participants who are prescribed antihypertensive medications at baseline.

\section{METHODS}

\section{Study design}

REACH is a two-parallel group, double-blind randomised controlled trial that will utilise a two (groups: e-counselling vs control) by three (assessment intervals: baseline, 4-month and 12-month outcome) design. Randomisation will be blocked within each of our four recruitment sites (Toronto, London, Grey Bruce region and Vancouver) to ensure overall balance $(1: 1)$ in the number of participants assigned to the two groups. This trial will be double blind according to CONSORT standards. ${ }^{15}$ The e-counselling and control groups will be informed that they will receive e-messages "...that are designed to support a heart healthy lifestyle which is an essential component of blood pressure management." Therefore, all participants will be unaware of whether their intervention is the 'experimental' treatment. A randomisation code will be embedded into each participant ID number, which is known only to the Research Coordinator but not to the Principle Investigators or research assistants who administer the assessments. Blinding will be maintained during all assessment sessions (baseline, 4-month and 12-month intervals) and during the data processing and analysis of clinical outcomes. Moreover, participants will be instructed to not discuss their e-based treatment during these assessments. Over the 12-month intervention, 28-30 e-based messages (dependent on \# weeks/month) will be sent to controls and e-counselling participants-see table 1. E-messages will be sent on a weekly basis during months $1-4$, then 
Table 1 Twelve-month schedule for pro-active e-messaging to e-counselling and control groups

\begin{tabular}{llll}
\hline Schedule: & Months 1-4=weekly & Months 5-8=bi-weekly & Months 9-12=monthly \\
\hline Number of e-messages & $17-18$ & $8-9$ & 4 \\
\hline
\end{tabular}

bi-weekly between months 5 and -8 , and then monthly between months 9 and -12 (figure 1).

\section{Inclusion/exclusion criteria}

Participants will be 35-74 years of age and diagnosed with stage 1 or 2 hypertension (SBP, 140-180mm Hg; DBP $90-110 \mathrm{~mm} \mathrm{Hg})$. Among participants not prescribed medication, hypertension status will be confirmed at baseline by a medical doctor or a nurse practitioner. Participants prescribed antihypertensive drugs will be required to have the prescription unchanged for at least 2 months before enrolment. In order to prevent 'floor effects' for the analysis of primary outcomes, participants prescribed antihypertensive medications will also be required to have a baseline $\mathrm{BP}$ that is at least in the high normal range $(\mathrm{SBP} \geq 130 \mathrm{~mm} \mathrm{Hg}$ and/or $\mathrm{DBP} \geq 85 \mathrm{~mm} \mathrm{Hg})$. Exclusion will be based on diagnosis of sleep apnoea, kidney disease, major psychiatric illness (eg, psychosis), alcohol or drug dependence in the previous year; pregnancy, institutional residence or an inability to comprehend English.

\section{Experimental versus control interventions Control}

An ethical imperative for any long-term trial of preventive e-counselling for hypertension is to ensure that the control arm receives essential information about preventive guidelines for BP management. From a methodological perspective, it is a priority to balance e-based support across the control and experimental arms of the trial to ensure that potential improvement in clinical outcome is not confounded by differences in nonspecific support and attention. Therefore, controls will be supported with educational material that will include brief articles on heart healthy living that are selected from the Resources section of the Heart and Stroke Foundation Blood Pressure Action Plan. The e-based articles for controls will provide information regarding appointments with physicians, BP measurement, lifestyle and antihypertensive medication.

\section{e-Counselling}

Participants will receive e-messages that will guide them in self-assessing their current stage of motivational readiness for adhering to self-management behaviours as a daily lifestyle routine. These participants will be directed to select their behaviour change priority according to the recommended goals for exercise, diet, smoke-free living and adherence to prescribed antihypertensive medications. In keeping with the guidelines from motivational interviewing, participants will be sent an ecounselling message that is tailored to their self-reported stage of readiness for change. Each message will provide a link to the e-counselling program on the Heart and Stroke Foundation website. A menu of stage-appropriate e-resources for lifestyle change will be presented which will include brief video-taped counselling messages that validated to each participant's presenting stage of readiness for change. This feedback will be supplemented by online self-monitoring charts and e-handouts that provide guidelines/tips for initiating or maintaining lifestyle change. The content of each video-taped message will be consistent with clinical guidelines for motivational interviewing, ${ }^{14}$ as established in our previously adapted manual. ${ }^{16}$ Accordingly, the video clips will highlight key clinical features which are not only applicable to our targeted self-management behaviours, but also promote the following: validation of the participant's presenting stage of readiness for behaviour change,

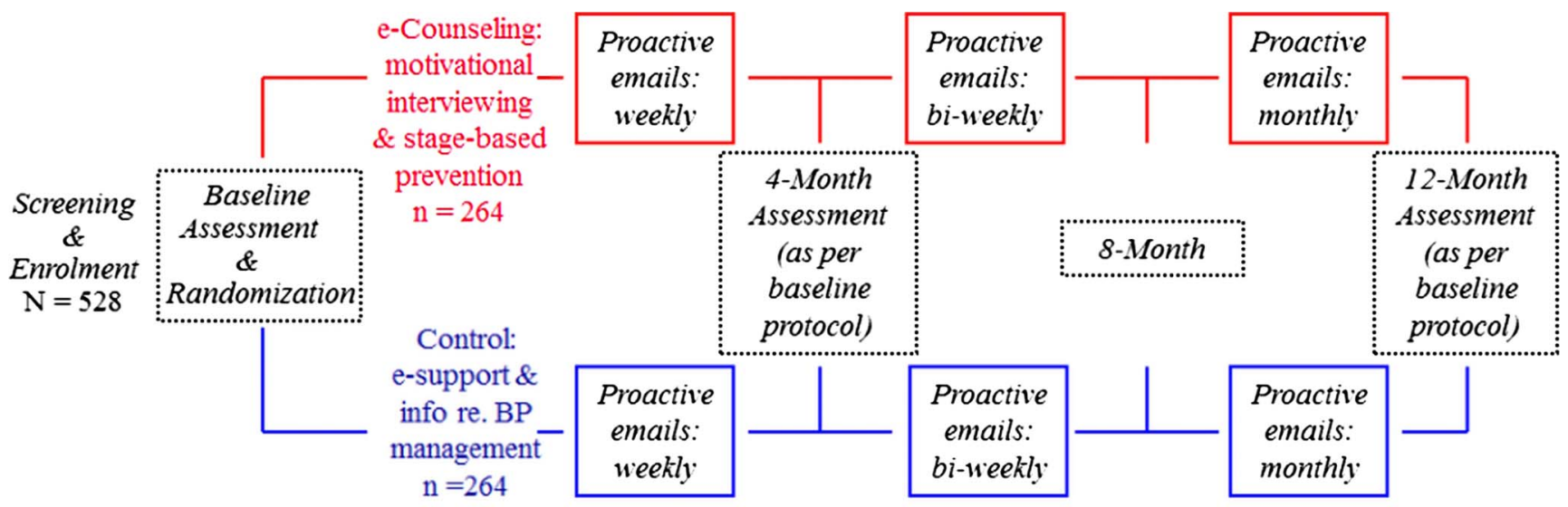

Figure 1 Schematic summary of reducing risk with e-based support for adherence to lifestyle change in hypertension. 
individual review of salient pros versus cons for change that determine decisional balance, resolution of ambivalence for change, increased efficacy for initiating change, and review of social and cognitive-behavioural skills for relapse prevention to maintain adherence.

\section{Recruitment and randomisation}

We will replicate the successful recruitment strategy from our previous trial (I-START) ${ }^{13}$ by informing online visitors to the Heart and Stroke Foundation Risk Assessment Program about our study. This message will only appear once participants confirm online that they reside in our participating sites within Canada: Toronto, London, the Grey Bruce region of Ontario and Vancouver. Eligible participants will then undergo clinical assessment at the local participating sites: (1) University Health Network, Toronto, (2) London Health Sciences Center, Univ. of Western Ontario, London, (3) Grey Bruce Health Unit, Owen Sound and (4) UBC, Vancouver Coastal Health, Vancouver. Recruitment will take place between February 2012 and July 2015. The Research Coordinator and Principal Investigator will be based in the Behavioural Cardiology Research Unit, University Health Network, Toronto and a Coordinator/Research Assistant will be based in each recruitment site.

Randomisation will be conducted by a computer program (randomize.net) using randomly permuted blocks to assign participants to control or e-counselling. The randomisation code will be hidden from research assistants during assessments and data processing of the primary and secondary outcomes. An unbiased response by participants to the REACH protocols will be facilitated by frequent and sustained e-messages (table 1) that are likely to be perceived as being supportive in nature.

\section{Assessment protocol}

Baseline, 4-month and 12-month assessments in REACH will be scheduled between 08:00 and 12:00 to minimise diurnal BP variability. Participants will be instructed to fast for $12 \mathrm{~h}$ in order to obtain a valid profile of lipoprotein cholesterol. They will also be directed to refrain from smoking for at least $4 \mathrm{~h}$, caffeine for $12 \mathrm{~h}$ and strenuous exercise for $24 \mathrm{~h}$ prior to their appointment. Anthropometric characteristics, medical history and medication information will be collected. Medication adherence will be calculated by the Medication Possession Ratio, which is defined as the quantity of antihypertensive medication that has been utilised over each 4-month interval versus the quantity that has been prescribed, based upon pharmacy refill data. ${ }^{17}$ Resting BP, physical activity, dietary behaviour, psychological distress and participant's readiness to change will be examined at each assessment interval. Feedback of the Framingham 10-year absolute risk for CVD will be provided to the participants at the end of each assessment.

\section{Blood pressure}

Primary outcome measures of SBP, DBP and PP will be assessed by a validated protocol for automated BP assessments using the BpTRU device. Participants will be seated for at least $5 \mathrm{~min}$ prior to activation of BpTRU. The BP cuff will be applied to the left arm of the participant by a nurse or trained research assistant. An initial BP measurement will be recorded. The nurse/research assistant will then exit the room while the BpTRU device completes an automated series of five BP recordings. A 1 min interval will separate each of these recordings. The recorded BP (SBP, DBP, PP) at each assessment interval will be the mean of these five BpTRU measurements.

\section{Diet}

Adherence to a sodium-restricted diet will be evaluated by $24 \mathrm{~h}$ urinary sodium analysis and defined as $\leq 100 \mathrm{mmol} /$ day. Adherence to recommended guidelines for daily intake of fruits, vegetables and supplementary dietary fat will be evaluated by the NIH/NCI DHQ. which has established validity ${ }^{18}$ and has been successfully adapted for a Canadian population ${ }^{19}$ and used in Canadian studies. ${ }^{20}$

\section{Physical activity/exercise}

Daily activity will be defined as the mean 4-day number of steps recorded on a tri-axial pedometer (LifeSource/ A\&D XL-18CN Activity Monitor). Adherence to physical activity will also be measured by a validated assessment: RPAQ. ${ }^{21}$ RPAQ measures self-reported time in activities (associated with light, moderate and intense energy expenditure) at home, work and recreational settings over the previous month.

\section{Salivary cotinine}

Physiological evaluation of smoking status will be recorded by salivary cotinine among participants who are current smokers at baseline. Positive exposure to nicotine, which is consistent with smoking, will be defined by cotinine $\geq 10 \mathrm{ng} / \mathrm{mL}$. This evaluation will screen for exposure to nicotine replacement therapy, which would confound the interpretation of the cotinine index.

\section{Readiness for change}

Prochaska's Transtheoretical algorithm will be used to operationally define motivational readiness to initiate or maintain self-management behaviours that include planned exercise, daily activities for active living, fruit and vegetable intake, salt use, alcohol consumption and smoke-free living. The stages of change are conventionally defined as follows: precontemplation (not ready to adhere to the target behaviour in the next 6 months), contemplation (ready to adhere to the target behaviour in the next 6 months), preparation (ready to adhere to the target behaviour in the next 4 weeks), action (adhere to the behaviour but for less than 6 months) and maintenance (adhere to the behaviour for 6 months or more). ${ }^{22}$ 


\section{Psychological distress}

Diagnostic screening tools will be used to assess anxiety and depression: Generalized Anxiety Disorder-7 and the Patient Health Questionnaire-9, respectively. Psychological stress will be assessed using the Perceived Stress Scale. The validity and reliability of these questionnaires are well established. ${ }^{23-25}$

\section{Sample size}

The sample size estimation was based on the meta-analysis by Neubeck $e t a l^{26}$ who evaluated the efficacy of randomised controlled trials of preventive telehealth and e-based programs in modifying BP: weighted mean difference in SBP between experimental versus control groups $=-4.69 \mathrm{~mm} \mathrm{Hg}$, common pooled variance $=354$, pooled $\mathrm{SD}=18.81$. In order to replicate this outcome, the estimated sample $=254$ participants per group (using a two-parallel group design, type 1 error $=5 \%$ and power $=80 \%$ ). Dropouts from this study will be interpreted as an intervention failure. Therefore, we will recruit a sample of 528 participants.

\section{Statistical analysis}

Linear Mixed Models with a random effects intercept will be used to evaluate whether within-participant change across assessment intervals is independently and significantly associated with the e-counselling group, for each primary and secondary outcome. ${ }^{27}$ Generalised estimation equations for repeated measures will be used to analyse categorical outcomes (eg, smoking cessation status). All significant interactions or main effects in our statistical models will be followed up with post hoc contrasts, using a Bonferroni correction for the statistical significance criterion of $\mathrm{p}<0.05$.

\section{Ethics and dissemination}

All participants will be provided written informed consent. Ethics approval will be renewed on an annual basis.

Meta-analytic evidence clearly supports the use of lifestyle counselling to reduce SBP and risk for premature mortality. Programs aimed at improving selfmanagement behaviours significantly decrease SBP by $3-8.7 \mathrm{~mm} \mathrm{Hg}$ relative to controls. ${ }^{8-10}$ In order to optimise the efficacy of lifestyle change interventions, it is essential to develop strategies to sustain long-term adherence to recommended self-management behaviours.

Once the long-term efficacy of preventive e-counselling is established as exemplified by this trial, it is likely that this strategy will become increasingly prominent in the development of community-based initiatives for CVD prevention. This point is highlighted by evidence that there is only low-to-moderate long-term adherence to recommended standards for exercise, diet and smokefree living following conventional behaviour change programs. ${ }^{28-30}$ Moreover, there is high potential to use e-counselling to increase long-term adherence to antihypertensive pharmacotherapy. Our team and others have reported that non-adherence to antihypertensive medication ranges from $15 \%$ to $50 \%,{ }^{17} 31$ while persistence with antihypertensive medications diminishes over time for approximately $22-50 \%$ of patients. ${ }^{31} 32$

Knowledge translation of findings obtained from this proposed trial will be utilised to guide the ongoing development of e-counselling services provided by the Heart and Stroke Foundation. This e-based service can be currently accessed through three portals: My Blood Pressure Action Plan, My Heart and Stroke Risk Assessment and My Healthy Weight Action Plan: http://www. heartandstroke.com/site/c.ikIQLcMWJtE/b.5374487/k. 62BF/HeartStroke_Action_Plans.htm.

Internet traffic is significant on this website. In 2011, 395044 individuals accessed this website for health information, while 160600 persons completed the Heart and Stroke Risk Assessment program.

In sum, we anticipate that findings from the REACH trial will have high population impact, given our collaboration with the Heart and Stroke Foundation of Canada. Advancements achieved with this trial, concerning the content and methodology of preventive e-counselling, will be widely disseminated to the general public and most likely utilised in follow-up research by our team and others.

\section{Author affiliations}

${ }^{1}$ Behavioural Cardiology Research Unit, University Health Network, Toronto, Ontario, Canada

${ }^{2}$ Faculty of Medicine, University of Toronto, Toronto, Ontario, Canada

${ }^{3}$ Departments of Medicine and of Physiology and Pharmacology, University of Western Ontario, Toronto, Ontario, Canada

${ }^{4}$ Faculty of Medicine, University of British Columbia, Vancouver, British Columbia, Canada

${ }^{5}$ Faculty of Land and Food Systems, University of British Columbia, Vancouver, British Columbia, Canada

${ }^{6}$ Grey Bruce Public Health Unit, Owen Sound, Ontario, Canada

${ }^{7}$ Faculty of Kinesiology and Physical Education, University of Toronto, Toronto, Ontario, Canada

${ }^{8}$ Toronto Rehabilitation Institute, Toronto, Ontario, Canada

${ }^{9}$ Department of Family Medicine, McGill University, Montreal, Quebec, Canada

${ }^{10}$ Department of Cardiology, University Health Network, Toronto, Ontario,

Canada

${ }^{11}$ London Health Science Center, London, Ontario, Canada

${ }^{12}$ School of Kinesiology, University of Western Ontario, London, London, Canada

Contributors RPN conceived the study. RPN, RF, MD, SB, HL, FG, ST, JG, $\mathrm{PO}, \mathrm{JK}, \mathrm{CC}, \mathrm{VH}$ and KS contributed to the study design. RPN, SL, RF, MD, FG and $\mathrm{HL}$ participated in conducting the trial and acquisition of data. RPN and SL drafted and revised the manuscript. All authors contributed critical intellectual input and approved the final manuscript.

Funding This trial is supported by the Canadian Institute of Health Research, grant number (MOP-111242).

Competing interests None.

Patient consent Obtained.

Ethics approval University Health Network, University of British Columbia, London Health Sciences Center and the Grey Bruce Public Health Unit.

Provenance and peer review Not commissioned; internally peer reviewed.

Data sharing statement Data will not be automatically shared or distributed in a public forum. However, data will be made available upon request on a case by case basis. 
Open Access This is an Open Access article distributed in accordance with the Creative Commons Attribution Non Commercial (CC BY-NC 3.0) license, which permits others to distribute, remix, adapt, build upon this work noncommercially, and license their derivative works on different terms, provided the original work is properly cited and the use is non-commercial. See: http:// creativecommons.org/licenses/by-nc/3.0/

\section{REFERENCES}

1. Sagie A, Larson MG, Levy D. The natural history of borderline isolated systolic hypertension. N Engl J Med 1993;329:1912-17.

2. Wilkins K, Campbell N, Joffres MR, et al. Blood pressure in Canadian adults. Health Rep 2010;21:37-46.

3. Leenen $\mathrm{FH}$, Dumais J, Mcinnis $\mathrm{NH}$, et al. Results of the Ontario survey on the prevalence and control of hypertension. CMAJ 2008;178:1441-9.

4. Lewington S, Clarke R, Qizilbash N, et al. Age-specific relevance of usual blood pressure to vascular mortality: a meta-analysis of individual data for one million adults in 61 prospective studies. Lancet 2002;360:1903-13.

5. Chobanian AV, Bakris GL, Black HR, et al. The seventh report of the joint national committee on prevention, detection, evaluation, and treatment of high blood pressure: the JNC 7 report. JAMA 2003;289:2560-72.

6. Campbell NR. The 2001 Canadian hypertension recommendations -what is new and what is old but still important. Can J Cardiol 2002;18:591-603.

7. Williams B, Poulter NR, Brown MJ, et al. British Hypertension Society Guidelines for Hypertension Management 2004 (BHS-IV): summary. BMJ 2004;328:634-40.

8. Hooper L, Bartlett C, Davey Smith G, et al. Reduced dietary salt for prevention of cardiovascular disease. Cochrane Database Syst Rev 2003;(3):Cd003656

9. Kelley GS, Kelley KS. Progressive resistance exercise and resting blood pressure: a meta-analysis of randomized controlled trials. Hypertension 2000;35:838-43.

10. Whelton SP, Chin A, Xin X, et al. Effect of aerobic exercise on blood pressure: a meta-analysis of randomized, controlled trials. Ann Intern Med 2002;136:493-503.

11. Statistics Canada. Canadian Internet Use Survey: 2007. In: Canada S, ed. June 2008. Ottawa, Ontario: Statistics Canada, 2008.

12. Nolan RP, Hachinski V, Wennberg R, et al. Therapeutic benefit of internet-based lifestyle counseling for hypertension. Vancouver, BC Canada: International society of hypertension, 2010.

13. Nolan RP, Liu S, Shoemaker JK, et al. Therapeutic benefit of internet-based lifestyle counselling for hypertension. Can J Cardiol 2012;28:390-6.

14. Miller WR, Rollnick S. Motivational interviewing: preparing people for change. 2nd edn. New York: Guilford Press, 2002.

15. Moher D, Hopewell S, Schulz KF, et al. Consort 2010 explanation and elaboration: updated guidelines for reporting parallel group randomised trials. BMJ 2010;340:C869.
16. Nolan RP. The Community Outreach Heart Health and Risk Reduction Trial: Facilitator's Guide. Toronto, Ontario, Canada: University Health Network, 2002.

17. Gwadry-Sridhar F, Guyatt G, O'brien B, et al. Teach: trial of education and compliance in heart dysfunction chronic disease and heart failure (HF) as an increasing problem. Contemp Clin Trials 2008;29:905-18.

18. Subar AF, Thompson FE, Kipnis V, et al. Comparative validation of the Block, Willett, and National Cancer Institute Food Frequency Questionnaires: the eating at America's table study. Am J Epidemiol 2001;154:1089-99.

19. Csizmadi I, Kahle L, Ullman R, et al. Adaptation and evaluation of the National Cancer Institute's Diet History Questionnaire and Nutrient Database for Canadian populations. Public Health Nutr 2007;10:88-96.

20. Bedford JL, Barr SI. The relationship between $24-\mathrm{H}$ urinary cortiso and bone in healthy young women. Int $J$ Behav Med 2010;17:207-15.

21. Besson $\mathrm{H}$, Brage $\mathrm{S}$, Jakes RW, et al. Estimating physical activity energy expenditure, sedentary time, and physical activity intensity by self-report in adults. Am J Clin Nutr 2010;91:106-14.

22. Prochaska JO, Velicer WF, Rossi JS, et al. Stages of change and decisional balance for 12 problem behaviors. Health Psychol 1994;13:39-46.

23. Spitzer RL, Kroenke K, Williams JB, et al. A brief measure for assessing generalized anxiety disorder: the Gad-7. Arch Intern Med 2006;166:1092-7.

24. Kroenke K, Spitzer RL, Williams JB. The PHQ-9: validity of a brief depression severity measure. J Gen Intern Med 2001;16:606-13.

25. Cohen J, Mannarino AP. Disseminating and implementing trauma-focused CBT in community settings. Trauma Violence Abuse 2008;9:214-26.

26. Neubeck L, Redfern J, Fernandez R, et al. Telehealth interventions for the secondary prevention of coronary heart disease: a systematic review. Eur J Cardiovasc Prev Rehabil 2009;16:281-9.

27. Verbeke G, Molenberghs G. Linear mixed models for longitudinal data. New York: Springer, 2000.

28. Barth J, Critchley J, Bengel J. Psychosocial interventions for smoking cessation in patients with coronary heart disease. Cochrane Database Syst Rev 2008;(1):Cd006886.

29. Myung SK, Mcdonnell DD, Kazinets G, et al. Effects of web- and computer-based smoking cessation programs: meta-analysis of randomized controlled trials. Arch Intern Med 2009; 169:929-37.

30. Young DR, Vollmer WM, King AC, et al. Can individuals meet multiple physical activity and dietary behavior goals? Am J Health Behav 2009:33:277-86.

31. Burnier M. Medication adherence and persistence as the cornerstone of effective antihypertensive therapy. Am J Hypertens 2006;19:1190-6.

32. Morgan SG, Yan L. Persistence with hypertension treatment among community-dwelling BC seniors. Can J Clin Pharmacol 2004;11: E267-73. 